\title{
Retrieving atmospheric turbulence information from regular commercial aircraft using Mode-S and ADS-B
}

\author{
Jacek M. Kopeć ${ }^{1,2}$, Kamil Kwiatkowski ${ }^{1,4,5}$, Siebren de Haan $^{3}$, and Szymon P. Malinowski ${ }^{2}$ \\ ${ }^{1}$ Interdisciplinary Centre for Mathematical and Computational Modelling, University of Warsaw, Warsaw, Poland \\ ${ }^{2}$ Institute of Geophysics, Faculty of Physics, University of Warsaw, Warsaw, Poland \\ ${ }^{3}$ Royal Netherlands Meteorological Institute, De Bilt, the Netherlands \\ ${ }^{4}$ Institute of Theoretical Physics, Faculty of Physics, University of Warsaw, Warsaw, Poland \\ ${ }^{5}$ University Centre for Environmental Studies and Sustainable Development, University of Warsaw, Warsaw, Poland \\ Correspondence to: Jacek M. Kopeć (jkopec@icm.edu.pl)
}

Received: 1 October 2015 - Published in Atmos. Meas. Tech. Discuss.: 16 November 2015

Revised: 12 April 2016 - Accepted: 20 April 2016 - Published: 23 May 2016

\begin{abstract}
Navigational information broadcast by commercial aircraft in the form of Mode-S EHS (Mode-S Enhanced Surveillance) and ADS-B (Automatic Dependent Surveillance-Broadcast) messages can be considered a new source of upper tropospheric and lower stratospheric turbulence estimates. A set of three processing methods is proposed and analysed using a quality record of turbulence encounters made by a research aircraft.

The proposed methods are based on processing the vertical acceleration or the background wind into the eddy dissipation rate. Turbulence intensity can be estimated using the standard content of the Mode-S EHS/ADS-B.

The results are based on a Mode-S EHS/ADS-B data set generated synthetically based on the transmissions from the research aircraft. This data set was validated using the overlapping record of the Mode-S EHS/ADS-B received from the same research aircraft. The turbulence intensity, meaning the eddy dissipation rate, obtained from the proposed methods based on the Mode-S EHS/ADS-B is compared with the value obtained using on-board accelerometer. The results of the comparison indicate the potential of the methods. The advantages and limitation of the presented approaches are discussed.
\end{abstract}

\section{Introduction}

Despite emergence of new forecasting techniques and availability of better forecasts the encounters of turbulence are the main cause of aviation incidents among commercial aircraft in the cruise phase (NTSB, 2011, 2012). The lack of onboard detectors that could provide appropriate warning for the pilot forces airmen to rely on forecasts, information from other nearby aircraft or traffic control or any slight visual characterizations (e.g. bands in cirrus Trier et al., 2010) of the presence of this otherwise invisible phenomenon. However, the scales of turbulence "detected" by the aircraft cause significant problems in forecasting. Most commercial aircraft is susceptible to turbulence on scales from $10 \mathrm{~m}$ up to $1 \mathrm{~km}$ (Lester, 1994). This is still below scale range modelled by the numerical weather prediction (NWP) models whose spatial resolution is in range from 1.5 up to $30 \mathrm{~km}$. Some trials have been made to use sub-grid-scale schemes of NWP models for direct estimation of turbulence (Trier et al., 2012). However, in order to give reasonable results, such approach requires resolutions higher than current operational standard. As of now most operationally used forecasting techniques infer clear-air turbulence (CAT) behaviour from larger scales resolved in the NWP models (e.g. Sharman et al., 2006; Gill and Buchanan, 2014). This methodology is based on widely accepted assumption that due to the turbulent energy cascade the presence (Wyngaard, 2010) of resolved scale turbulence is implying the presence of sub-scale turbulence. However, NWP-based forecast is bound to generate errors and requires sophisticated statistical corrections (Williams, 2014; Sharman et al., 2006; Kim et al., 2015).

Aside from forecasting, CAT is a broad field of research (Sharman et al., 2012) as its presence is determined by complex mechanisms accompanying hydrodynamical instabil- 
ities. The basic generation mechanisms include buoyancy waves breaking, flow over complex topography, presence of deep convection, and significant wind shears with KelvinHelmholtz instabilities. All of these mechanisms are responsible for the presence of turbulence in the upper troposphere and lower stratosphere (UTLS) that is hardly visible, except for the cases where the flow disturbs nearby cirrus clouds. At the same time systematic detection of CAT using the conventional weather radars is impossible because these radars are able to detect turbulence either in the presence of appropriate tracers (e.g. water droplets, ice crystals) or in the clear air provided the sufficient variation in the refractive index occurs. The former method is not applicable to CAT while the latter has been found to be problematic in some cases (James, 1980). The only feasible remote detection method is by using active optical sensing (e.g. forward-looking on-board lidar) (Vrancken, 2015). On-board devices allowing this are now in very early stages of development. Presently the most accurate method of measuring CAT is a dedicated airborne experiment (Koch et al., 2005; Veerman et al., 2014). However, in order to gain satisfactory information about such a complex phenomenon a large number of those expensive, time-consuming experiments would be required. Other sources of information are the standard turbulence reports provided by the commercial aircraft (PIREP, AMDAR/ACARS). For a long time investigation of kilometre-scale turbulence dynamics based on those reports was limited due to their sparse spatial resolution. The situation changed with introduction of in situ eddy dissipation rate $\left(\epsilon\right.$; notation used: $\left.\mathrm{EDR}=\epsilon^{\frac{1}{3}}\right)$ measurements (Cornman et al., 1995) with reporting resolution of order of $10 \mathrm{~km}$. The new resolution allowed for in-depth investigation of near-cloud turbulence and new insight into its occurrence (Trier et al., 2010; Trier and Sharman, 2009). The only drawback of the EDR data is that they are still not in widespread use, and their availability is by negotiation with individual airlines. This in turn severely limits the extent and volume of the available data.

In the present paper we propose new methods of gaining information about turbulence from regular commercial aircraft and demonstrate its viability. The proposed methods are based on a non-standard source, namely the Mode-S EHS (Enhanced Surveillance) data. The Mode-S EHS is a part of secondary surveillance radar (SSR) - a system supporting air traffic management (ATM) - and to our best knowledge it was not considered a source of turbulence measurements previously. Mode-S is a protocol of information exchange between the aircraft and the ATM system, whereas Mode-S EHS is a subclass of messages exchanged using this protocol that is of interest in the present study. It is based on the exchange of the short status messages or requests. The messages that are an integral part of the Mode-S are exchanged with a very high sampling rate ranging from 0.2 up to $2 \mathrm{~Hz}$ (ICAO, 2007b, 2004). The signal is transmitted using radio frequencies $1090 \mathrm{MHz}$ (downlink) and $1030 \mathrm{MHz}$ (uplink). The messages are not encrypted and thus can be received and decoded by relatively simple hardware (Haan et al., 2013; Stone and Pearce, 2016). It has been shown (Haan, 2011) that very high-frequency wind information can be derived from the Mode-S EHS. The methods we propose use this high-frequency measurement and the position information in order to perform turbulence measurements with spatial resolution of approximately $25 \mathrm{~km}$ at the cruising speed of $250 \mathrm{~m} \mathrm{~s}^{-1}$ which is approximately 1.6 of the in situ EDR data spatial resolution (approximately $15 \mathrm{~km}$ at $250 \mathrm{~m} \mathrm{~s}^{-1}$ ).

We also propose a method to extract turbulence information from a class of navigational messages named $\mathrm{Au}-$ tomatic Dependent Surveillance-Broadcast (ADS-B). ADS$\mathrm{B}$ are $1090 \mathrm{MHz}$ band messages automatically transmitted by the aircraft and formatted in the manner similar to the Mode-S. The ADS-B messages do not contain wind information and are broadcast with sampling rate between 1 and $6 \mathrm{~Hz}$. We know of one other alternative approach to ADSB being investigated simultaneously (Krozel and Sharman, 2015).

The potential advantage of proposed methods over the in situ EDR is the significantly larger volume of available information since very significant part of the commercial civil aviation aircraft worldwide use the Mode-S protocol. Especially taking into account the fact that the setting up of Mode-S/ADS-B receiving station requires relatively low amount of work and is relatively inexpensive. It is worth keeping in mind that in the situ measurements will probably be always more accurate, and thus the Mode-S EHS/ADS-Bbased turbulence estimates should be treated as a useful supplement to the measurements originating from the on-board devices rather than a replacement.

\section{Data used for analysis}

\subsection{Data set details}

It is hard to find a turbulence encounter for which both a good scientific-grade recording and the archive of the Mode-S EHS data are available at a same time. We managed to identify a record of the Mode-S EHS messages broadcast by a research aircraft during a flight campaign targeted explicitly at CAT measurements (Veerman et al., 2014). The Mode-S EHS record was received and stored by the Royal Dutch Meteorological Institute (KNMI). The KNMI data were collected using a locally installed receiver with spatial range marked in Fig. 1. Due to this limitation there is no overlapping record of turbulence encounter but there are numerous overlapping flight fragments allowing for proper analysis based on the synthetic Mode-S EHS and ADS-B messages generated using flight campaign data set. The procedure leading to generation of the synthetic Mode-S EHS/ADS-B messages can be defined and verified using the overlapping flight fragments. 


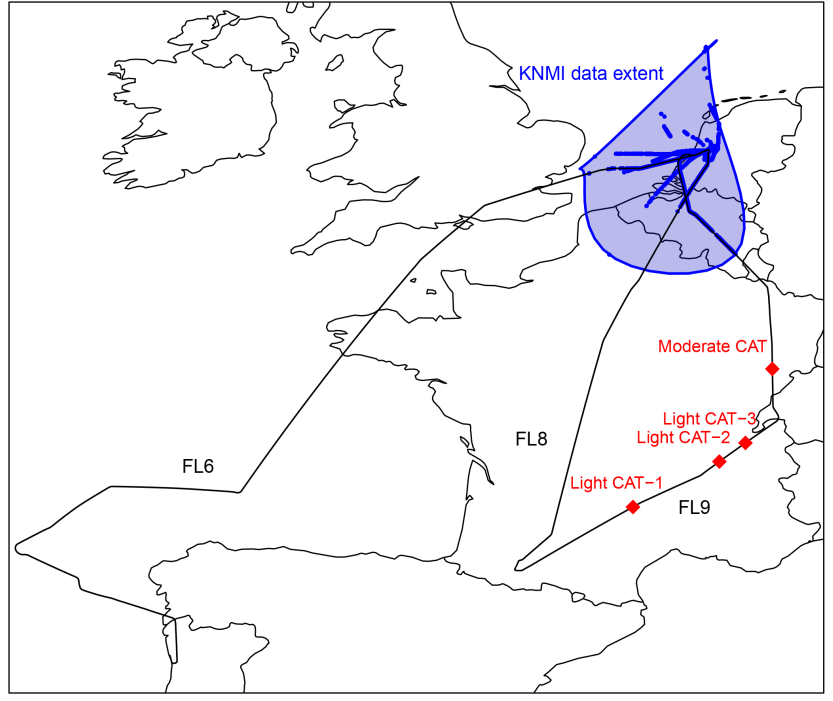

Figure 1. Tracks of DELICAT flights FL6, FL8 and FL9 (black solid lines). Peak turbulent events (red markers and red caption). KNMI data (blue dots) and KNMI data range (light blue shape).

The KNMI data set comprises mixed ADS-B and Mode-S EHS messages. For details on a way the messages are captured, interpreted, processed, and stored see Haan et al. (2013). The available overlapping data are characterized by varying level of quality and availability. Summary of the KNMI data content can be found in Table 1. For a technical reason part of Mode-S EHS messages containing the true track angle parameter (ground track direction with respect to true north) of the research aircraft were missing from KNMI archive.

Since the KNMI data lack turbulence encounters and some essential parameters (low availability of velocities and lack of track angle), the analyses conducted in this paper are mainly based on a data set originating from flight measurement campaign conducted as a part of EU Seventh Framework Programme project DEmonstration of LIdar based Clear-Air Turbulence detection (DELICAT) (Veerman et al., 2014). The campaign aimed to test the feasibility of using a forward-looking lidar system as an early warning turbulence detector which was the main aim of the DELICAT project itself. The aircraft used for flight tests was a modified Cessna 550 Citation II aircraft belonging to National Aerospace Laboratory, Netherlands (NLR). The aircraft was equipped with the mentioned forward-looking lidar system, nose boom for side-slip and angle of attack measurements, and Inertial Reference System (IRS) equipped with a 3-D accelerometer and Digital Air Data Computer (DADC). Eleven flights were conducted in the period between 17 July and 13 August 2013. The data types and sources used for the analyses presented later are listed in Table 2.

For further analysis we have chosen three flights designated as FL6, FL8, and FL9. Flight FL6 was conducted on
Table 1. Parameters and basic characteristics of the KNMI data used in this paper. Nominal reporting frequency is based on whether the parameter is ADS-B or Mode-S EHS content. Data availability is a percent of all aircraft reports where the parameter was successfully received and decoded.

\begin{tabular}{llrr}
\hline Data type & Data resolution & $\begin{array}{r}\text { Nominal } \\
\text { sample rate }\end{array}$ & $\begin{array}{r}\text { Data } \\
\text { availability }\end{array}$ \\
\hline Longitude & $0.00024^{\circ}$ & $>1 \mathrm{~Hz}$ & $100.0 \%$ \\
Latitude & $0.00002^{\circ}$ & $>1 \mathrm{~Hz}$ & $100.0 \%$ \\
Ground speed & $2 \mathrm{kt}\left(1.03 \mathrm{~m} \mathrm{~s}^{-1}\right)$ & $>1 \mathrm{~Hz}$ & $5.6 \%$ \\
True airspeed & $2 \mathrm{kt}\left(1.03 \mathrm{~m} \mathrm{~s}^{-1}\right)$ & $0.2 \mathrm{~Hz}$ & $5.6 \%$ \\
Altitude & $25 \mathrm{ft}(7.62 \mathrm{~m})$ & $>1 \mathrm{~Hz}$ & $99.6 \%$ \\
Vertical rate & $64 \mathrm{ft} \mathrm{min}-1$ & $>1 \mathrm{~Hz}$ & $99.7 \%$ \\
& $\left(0.32 \mathrm{~m} \mathrm{~s}^{-1}\right)$ & & \\
Heading & $0.1757^{\circ}$ & $0.2 \mathrm{~Hz}$ & $52.1 \%$ \\
\hline
\end{tabular}

Table 2. Parameters of DELICAT data used in this paper and their respective data sources.

\begin{tabular}{lccc}
\hline Data type & $\begin{array}{c}\text { Data } \\
\text { source }\end{array}$ & $\begin{array}{r}\text { Sample } \\
\text { rate }\end{array}$ & Resolution \\
\hline Longitude & IRS & $5 \mathrm{~Hz}$ & $0.000172^{\circ}$ \\
Latitude & IRS & $5 \mathrm{~Hz}$ & $0.000172^{\circ}$ \\
True airspeed & DADC & $8 \mathrm{~Hz}$ & $0.032 \mathrm{~m} \mathrm{~s}^{-1}$ \\
Baro corrected altitude & DADC & $16 \mathrm{~Hz}$ & $0.3 \mathrm{~m}^{-1}$ \\
Vertical rate & DADC & $16 \mathrm{~Hz}$ & $0.08 \mathrm{~m} \mathrm{~s}^{-1}$ \\
Track angle & IRS & $20 \mathrm{~Hz}$ & $0.0055^{\circ}$ \\
Ground speed & IRS & $10 \mathrm{~Hz}$ & $0.06 \mathrm{~m} \mathrm{~s}^{-1}$ \\
Heading & IRS & $20 \mathrm{~Hz}$ & $0.0055^{\circ}$ \\
\hline
\end{tabular}

6 August 2013 while the two remaining flights took place on 8 August 2013. Flights FL6 and FL8 were first of all selected because they overlap with a relatively long Mode-S EHS/ADS-B records by KNMI. No turbulence was encountered during those flights but turbulence was reported by other aircraft in the vicinity during FL8. Flight FL9 was selected because of multiple turbulence encounters of light to borderline moderate (peak vertical acceleration $0.4 \mathrm{~g}$ ) intensity as shown in Figs. 1 and 2. The data from the forwardlooking lidar served to confirm that the investigated encounters were in the clear air. However, it is not clear whether the encounters were CAT or a near-cloud turbulence according to classification introduced by Sharman et al. (2012). This choice of flights allows the analysis to be based on three different cases: turbulent flight (FL9) for validity check, calm flight for (FL6) sanity check, and calm flight with turbulence reported in vicinity (FL8) for detection precision check. The reference EDR has been calculated according to the method outlined in (WMO, 2003) based on the high-frequency reading of the on-board accelerometer. 

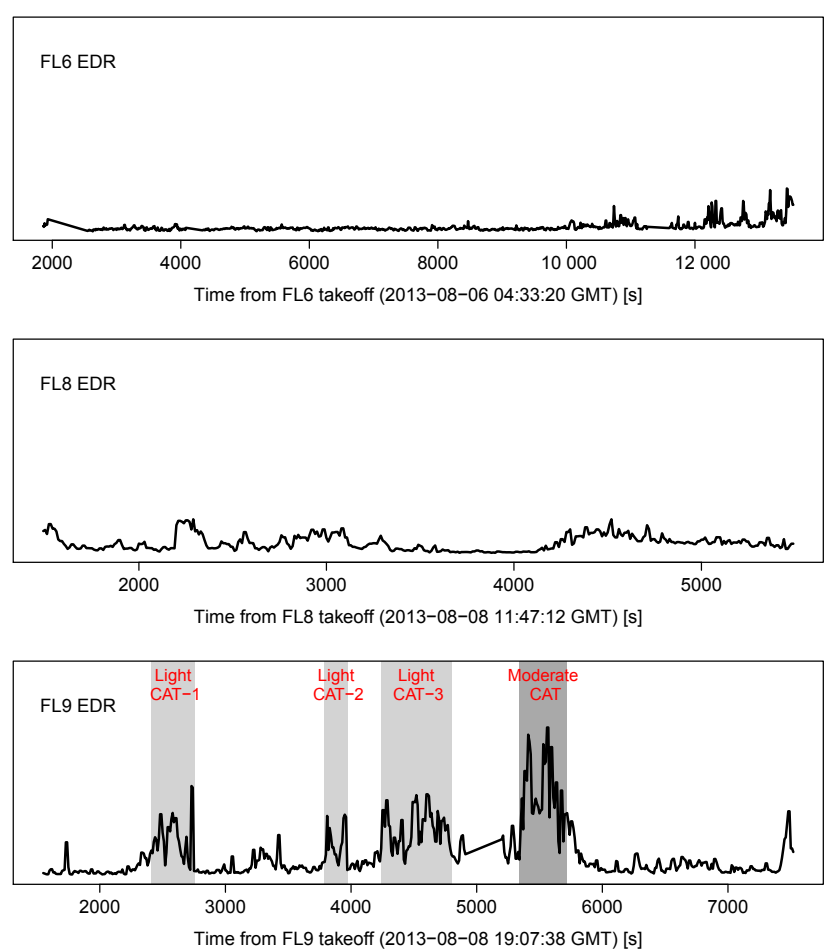

Figure 2. Reference EDR recordings from the DELICAT flights FL6, FL8, and FL9. Plots show $15 \mathrm{~s}$ maxima of the EDR in the cruise fragments of the flights. Vertical scale is arbitrary (yet the same on all plots) as EDR is scalable by a constant. Shaded areas in FL9 pane are the CAT occurrences (as in Fig. 1).
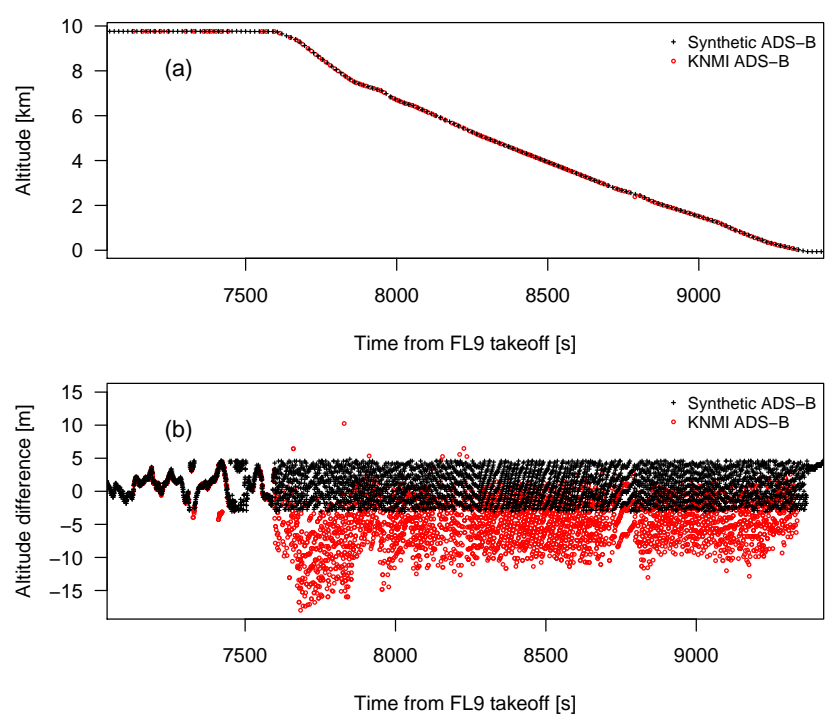

Figure 3. A comparison between the synthetic ADS-B data (black crosses) and reference KNMI ADS-B data (red circles) of (a) the altitude profile (note that for clarity $97 \%$ of the observations are not plotted here) and (b) the difference of the altitude with respect to the DELICAT reference altitude.

\subsection{Data set discussion}

In order to evaluate the appropriateness of the methods proposed in this paper we need to apply them to the Mode-S EHS data covering some turbulence encounters. Unfortunately no record of the original Mode-S EHS transmissions of the research aircraft was preserved that would include any turbulence encounter. Therefore we processed the highquality record of the DELICAT flight parameters of the aircraft in order to obtain an output that is intended to mimic the data sent by the Mode-S transponder. This processing was performed taking into account the technical parameters of the Mode-S protocol (including data sources, resolution, sampling rate, etc.) outlined in ICAO (2007b, 2004); Grappel and Wiken (2007). The original Mode-S data recorded by KNMI were used as a reference for evaluating the success of generating synthetic Mode-S EHS on the overlapping flight fragments.

The processing allowing for the DELICAT data to mimic the transponder output was quite straightforward.

1. For the reference KNMI data the chosen sampling rate was on average $1.5 \mathrm{~Hz}$ for flight 9 and $1.25 \mathrm{~Hz}$ for flight 8 due to data loss on reception. When the largest (few second) gaps are discarded the average sampling rate for both cases goes up to approximately $2 \mathrm{~Hz}$. This number was chosen for preparation of the ADS-B imitation. For the Mode-S EHS imitation we have chosen $0.25 \mathrm{~Hz}$, which corresponds to the typical rotation speed of the SSR antennas (ICAO, 2007c). We have also disregarded data loss in the present analysis since the fraction of reference data displaying greater than the assumed sampling rate did not exceed $5.6 \%$ for any flight.

2. The data contained in one sampling period are averaged. The averaged data are then discretized to match the reference data resolution summarized in Table 1. Discretization is performed using simple floor function. The resulting spatial resolution is $125 \mathrm{~m}$ for synthetic ADS-B and $1 \mathrm{~km}$ for synthetic Mode-S EHS (assuming the aircraft velocity is $250 \mathrm{~m} \mathrm{~s}^{-1}$ ).

The resulting data can be divided into three groups based on the agreement with the Mode-S EHS/ADS-B messages received in KNMI.

1. Very good agreement between the reference and the synthetic data as they are presented in Fig. 3. The DELICAT data originate from the DADC which is also the source for the Mode-S transponder.

2. Very good agreement but data are shifted by some nearly constant bias. This is valid for 2-D position (approximately $2 \mathrm{~km}$ horizontal shift as shown in Fig. 4) and heading (approximately $2^{\circ}$ bias). Those differences are small and almost constant, thus not distorting the results in any significant way. Data were accepted as correct. 


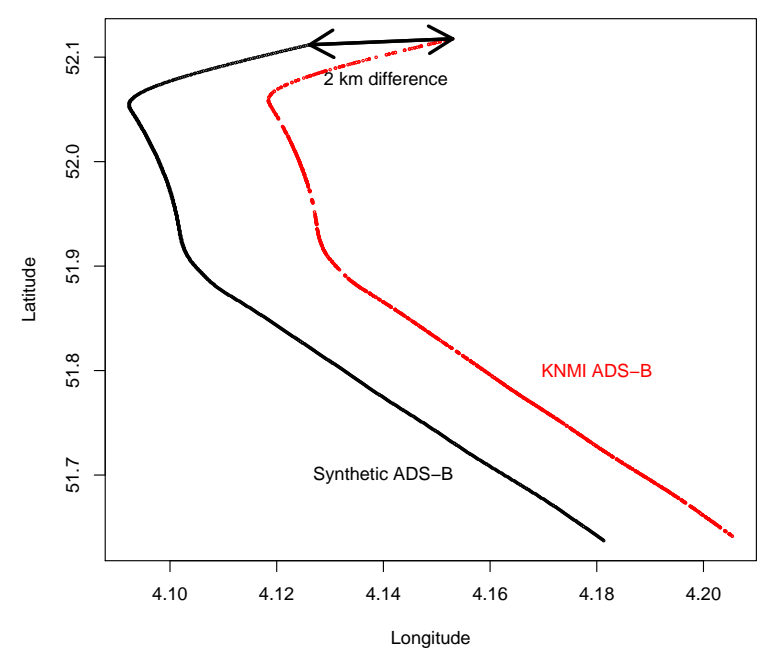

Figure 4. Comparison of flight paths as recorded by the KNMI receiver (red dots) and resulting from the processing of the DELICAT data (black dots) for a fragment of FL9. The $2 \mathrm{~km}$ error can be attributed to IRS drift which is of the order of $3 \mathrm{~km}$ per hour. IRS was calibrated prior to each flight.

3. Large shift in data noticeably changing over time: air speed and ground speed (both biased by $25 \mathrm{~m} \mathrm{~s}^{-1}$ ). This is despite the fact that the data source was the same.

The first two groups indicate that the processing algorithm is satisfactory, even though the 2-D position (longitude and latitude) reported in the DELICAT research data is not as accurate as that reported in the ADS-B/Mode-S EHS. However, the existence of third group might be bothersome. We have cross-checked the consistency of the ground speed record with the reported changes in the geographical position of the aircraft reported both in the KNMI and the DELICAT data. Although such estimate introduces additional errors it was clear that the ground speed recorded in KNMI was inconsistent with any of the three other ground speed estimates as it is presented in Fig. 5 (for the case of FL8). This inconsistency and the fact that velocities were reported in a very small fraction of data and in short interrupted periods indicate a possibility of systematic errors in reception.

In the case of the altitude we have discovered that in the ascent/descent phases of flight the agreement between the data received by the KNMI and the DELICAT data is worse than in the cruise phase (usually altitudes exceeding $7.5 \mathrm{~km}$ ), but these errors were relatively small. It was approximately 3 $18 \mathrm{~m}$ when vertical velocity was high and dropped to less than $6 \mathrm{~m}$ at the cruise altitudes when the flight was stable. Moreover, the error dominant sign was dependent on whether the aircraft was descending or ascending (negative for descend as shown in Fig. 3), which suggests the existence of a small time lag between the DELICAT and KNMI records rather than necessarily an error of this magnitude.

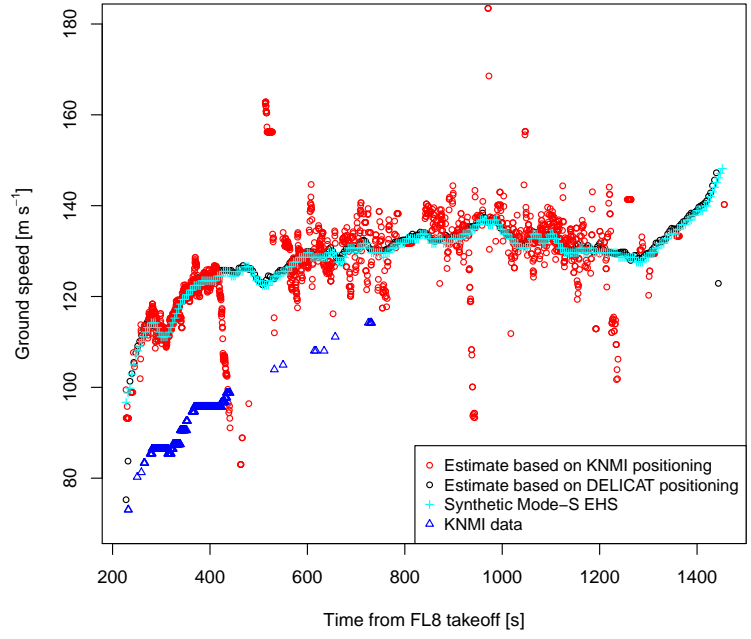

Figure 5. Comparison of ground speed record against estimates based on reported geographic position for the case of FL8. FL8 is presented because of the longest continuous KNMI record containing ground speed.

In summary, the reference data collected by the KNMI receiver proved not sufficient enough to be the sole basis for estimating EDR. This is mainly due to lack of the true track angle record and poor quality of existing speed records. The overlap regions also contain mainly ascent/descent and none of the DELICAT turbulence encounters. To mitigate this we have established a method of generating synthetic Mode-S EHS and ADS-B data based on DELICAT measurements. The output of this processing was verified against the reference KNMI data and deemed satisfactory.

\section{CAT estimation algorithms}

In the following section we propose three methods to process the Mode-S EHS and ADS-B data into potentially useful information. The first of those methods uses only the data available in ADS-B automatic transmissions, which guarantees independence from the presence of the ground-based interrogators. This increases the reception coverage significantly. The second and third methods require more information and hence a full set of Mode-S EHS data.

\subsection{Method 1: vertical acceleration}

The usual indicator of CAT encounter is the magnitude of the vertical acceleration of the aircraft. This is effectively what the passengers feel and what is reported in the simplest AMDAR messages containing the so-called Index of Turbulence. However, the value of the peak acceleration as measured by the aircraft poorly reflects the state of the background atmosphere (WMO, 2003). This is mainly due to the fact that various aircraft are characterized by vastly different aerodynamic properties, which leads to large variation in response to the 
vertical gusts characteristics. Moreover, neither Mode-S EHS nor ADS-B contains information about acceleration itself, yet they both carry altitude and vertical velocity information and, in the case of ADS-B transmissions, the reporting frequency is between 0.5 and $1 \mathrm{~Hz}$. Using this information the vertical acceleration of the aircraft can be calculated using the simple formulas:

$$
\begin{aligned}
& \frac{\mathrm{d} z}{\mathrm{~d} t}=v_{z}, \\
& \frac{\mathrm{d} v_{z}}{\mathrm{~d} t}=a_{z} .
\end{aligned}
$$

Here $z$ is the altitude, $v_{z}$ is the vertical velocity, and $a_{z}$ is the vertical acceleration. The time derivative is approximated simply as the finite difference between the consecutive observations. The $a_{z}$ obtained in this way is then processed as described in WMO (2003) in order to obtain the EDR estimate. This method employs a simple formula which models the aircraft response to the homogeneous, isotropic turbulence spectrum.

$\epsilon=B \sigma_{T}^{3} V^{-1}$

Here $B$ is an adjustable constant accounting for the unknown responsiveness factor of the aircraft, $\sigma_{T}$ is the standard deviation of acceleration measurements in a set period of time $T$, and $V$ is the mean true air speed (TAS) of the aircraft in the time period $T$.

Another way to salvage the same formulas (Eqs. 1-3) is to use the inertial vertical velocity (IVV) information which is present in the ADS-B. It also has a coarse resolution of $32 \mathrm{ft} \mathrm{min}^{-1}\left(0.16 \mathrm{~m} \mathrm{~s}^{-1}\right)$. The potential advantage is that since we start with an estimate of vertical velocity we can use first-order finite differences to get an estimate of the vertical acceleration. In Sects. 4 and 5 we present and discuss results from both approaches: IVV and altitude-derived EDR, called IVV EDR and ALT EDR respectively. Although this CAT estimation method is based solely on ADS-B, which extends the coverage, it has a number of drawbacks. First of all the ADS-B data do not contain TAS; therefore there are three possible ways to use the above formula:

- combine the information from Mode-S EHS about TAS with high resolution altitude and vertical rate - in this way, however, we lose the advantage of territorial coverage;

- assume constant TAS (which is not a particularly good approximation) and use only ADS-B;

- use an external wind information from, for example, the NWP model to recalculate TAS from the ground track. It will introduce an uncertainty related to the external wind source (see e. g. Mulally and Anderson, 2011). This option will not be discussed here.
For further analysis we have assumed constant TAS to evaluate purely ADS-B-based EDR estimation. We have also compared the results against the first approach - combined ADS-B and Mode-S EHS.

Another factor lowering effectiveness of this method is reporting the resolution of the altitude in the ADS-B, which is $25 \mathrm{ft}(7.62 \mathrm{~m})$. This causes the acceleration signal based on such an altitude profile to be erroneous and unrealistic. In order to mitigate this, prior to processing we have applied a second-order Butterworth band-pass filter (signal developers, 2013) with limiting frequencies 0.025 and $0.050 \mathrm{~Hz}$. Assuming that an typical commercial jet aircraft is cruising at $250 \mathrm{~m} \mathrm{~s}^{-1}$ these frequencies correspond to the spatial scales of 10 and $5 \mathrm{~km}$ respectively. The scale range below $5 \mathrm{~km}$ was dominated by the quantization noise. Analogous filtering was applied to IVV data.

Finally, there is one more potential issue that this method may display that will not be discussed in the present paper since all data presented here are derived from only one aircraft. Equation (3) is an approximation since in WMO (2003) $B$ incorporates the aircraft response integral, which may have very diverse values for various aircraft types. Basically this integral depends on some basic aircraft characteristics (wing area, mass, lift, etc.) and the flight conditions (TAS, the air density) (Wright and Cooper, 2014). Treating $B$ as a constant means holding the aircraft type, TAS, and air density as constant too. This will require very cautious use when applied to the data derived from the aircraft of varying type.

\subsection{Method 2: the structure functions}

The second proposed method aims to retrieve characteristics of the turbulence independently of the aircraft type. To achieve this goal we will employ a method of estimating structure functions value based on high-frequency wind velocity measurements. The structure function estimate is obtained as follows (Frehlich and Sharman, 2010):

$D_{\mathrm{LL}}(r)=\left\langle\left(u_{\mathrm{LL}}(\boldsymbol{x})-u_{\mathrm{LL}}(\boldsymbol{x}+\boldsymbol{r})\right)^{2}\right\rangle_{\boldsymbol{x}}$.

Here $u_{\mathrm{LL}}$ denotes the component of the horizontal wind velocity vector along the track of the aircraft, $\boldsymbol{x}$ is a position of measurement, $\boldsymbol{r}$ is the displacement vector, $r=|\boldsymbol{r}|$ is the displacement distance, and $\langle\cdot\rangle_{\boldsymbol{x}}$ stands for position and ensemble averaging. In order to estimate $D_{\mathrm{LL}}$ we have used a standard approach to aircraft originating time series described in Cho et al. (2003) and Meischner et al. (2001). However, in our analysis we deal with only the horizontal component of wind. That denies us the possibility to calculate $D_{\mathrm{LL}}$ accurately during aircraft turns. In addition, this method has been previously applied to time series with sampling rate $\mathrm{O}(10 \mathrm{~Hz})$, whilst in our case the sampling rate is $0.25 \mathrm{~Hz}$. Frehlich and Sharman (2010) have used similar methodology for estimating $D_{\mathrm{LL}}$ based on AMDAR measurements with even greater spatial distances than in our case (up to $1000 \mathrm{~km}$ ). However, their treatment of ensemble and 
spatial averaging is closer to the original Monin and Yaglom (1975) where ensemble average as an average over many realizations of the similar conditions.

It is then assumed that the structure function estimate $D_{\mathrm{LL}}(r)$ can be expressed as

$D_{\mathrm{LL}}(r)=C \epsilon^{\frac{2}{3}} r^{\frac{2}{3}}$.

Here $C$ is a constant. The theoretical value of $C$ is close to 2 (Sreenivasan, 1995). In order to obtain $\epsilon$ one needs to combine formulas 4 and 5 . This allows us to estimate the magnitude of EDR based on regressing $\log D_{\mathrm{LL}}(r)$ against $\log r$. In our case the separation can only take values from a discrete range determined by the intervals between the consecutive Mode-S EHS messages broadcasting. Those values are very sparsely distributed in the range up to $10 \mathrm{~km}$, where according to Frehlich and Sharman (2010) the structure function scaling is present. Typically observations are separated by approximately $0.8-1.1 \mathrm{~km}$, which results in 9-12 different $r$ values for the mentioned scale range. In practice this sparsity can be even greater due to receiver loss factor. In addition to this sparse distribution, the estimates of $D_{\mathrm{LL}}$ are impacted by effects of quantization noise in source data. This requires us to again use the second-order Butterworth filter. Since in the case of wind the quantization noise is not as significant we have chosen a frequency range covering the expected CAT scales. The limiting frequencies were chosen to be 0.025 and $0.094 \mathrm{~Hz}$, which corresponds to spatial scale between 10 and $2.6 \mathrm{~km}$ respectively.

\subsection{Method 3: threshold crossing}

The last method we present is also based on high-resolution horizontal wind measurements. However, this time we will employ a method described in Poggi and Katul (2010). The method is based on relating a density function of threshold crossing $\left(N_{\mathrm{d}}\right)$ to the eddy dissipation rate. For a series of $N$ consecutive measurements of longitudinal velocity component at discrete times $t_{i}, i \in\{1, \ldots, N\}$, the density of threshold crossing is defined as follows:

$N_{\mathrm{d}}\left(T_{c}, N\right)=\frac{\sum_{i=0}^{N} I\left(t_{i}, T_{c}\right)}{N-1}$.

Here $T_{c}$ is a non-dimensional threshold (positive or negative) and the indicator function $I\left(t_{i}, T_{c}\right)$ is defined as follows:

$I\left(t_{i}, T_{c}\right)=$
$\begin{cases}1 & \text { if }\left[\frac{u_{\mathrm{LL}}\left(t_{i}\right)}{\sigma_{u}}-T_{c}\right]\left[\frac{u_{\mathrm{LL}}\left(t_{i+1}\right)}{\sigma_{u}}-T_{c}\right]<0 . \\ 0 & \text { otherwise }\end{cases}$

Here $\sigma_{u}$ denotes the local root mean square of the longitudinal velocity fluctuations $u_{\mathrm{LL}}$. Following Poggi and Katul (2010), the threshold crossing density can be related to $\epsilon$ using

$\epsilon=\frac{15 \pi^{2}}{2} v \sigma_{u}^{2} e^{T_{c}^{2}} N_{\mathrm{d}}^{2}\left(T_{c}, N\right)$.
Here $v$ denotes the kinematic viscosity. For calculations in this paper we have taken $v$ to be the kinematic viscosity at the altitude of $11 \mathrm{~km}$ as defined by the US Standard Atmosphere (NASA, 1976). In our setting the wind velocity estimate is first filtered before passing to the threshold-crossing-based estimator. The filtering serves to remove both the mesoscale tendencies and high-frequency noise. For this purpose we have used again the second-order Butterworth band-pass filter. The filter limiting frequencies were 0.025 and $0.094 \mathrm{~Hz}$, which correspond roughly to 10 and $2.6 \mathrm{~km}$ respectively.

\subsection{Methods: summary}

We consider all three methods here because of their possible varying application. The first method uses only the vertical position (or vertical velocity) information available in ADSB. This makes it possible to receive data globally since ADS$\mathrm{B}$ is not limited to any areas. The main disadvantage of this method is its obvious dependence on the aircraft type. The second and the third methods (structure functions and threshold crossing) need high-resolution horizontal wind measurements. Therefore, their use is limited by territorial extent of Mode-S EHS data availability. This in turn is determined by the presence of the ground-based interrogators (implying e.g. lack of satisfactory ocean and sea coverage). However, they do not explicitly incorporate aircraft dependent variables into calculations, which reduces the potential aircraft type dependence.

The most interesting question is whether the available time resolutions of the measurements (approximately $4 \mathrm{~s}$ for Mode-S EHS and up to $0.5 \mathrm{~s}$ for ADS-B) will allow for the successful extraction of turbulence information.

\section{Results}

For each flight we have calculated a set of four EDR estimations:

1. ADS-B method based on inertial vertical velocity (IVV EDR)

\section{ADS-B method based on altitude (ALT EDR)}

3. Mode-S EHS-based structure function method

4. Mode-S EHS-based threshold crossing method.

The threshold crossing EDR (according to Eq. 8) as well as the structure function-based EDR is known exactly (when assuming the theoretical value of $C=2$ in Eq. 5). However, the results of ADS-B-based methods along with the reference DELICAT EDR are determined up to a constant $B$ (see Eq. 3). For the sake of presentation we have chosen an arbitrary value of $B$ for each time series so that their results ranges correspond to those obtained from Mode-S EHSbased methods. These values of $B$ are indicated in Figs. 6-8. In theory these constants should be the same for all methods. In practice the filtering and quantization are expected 


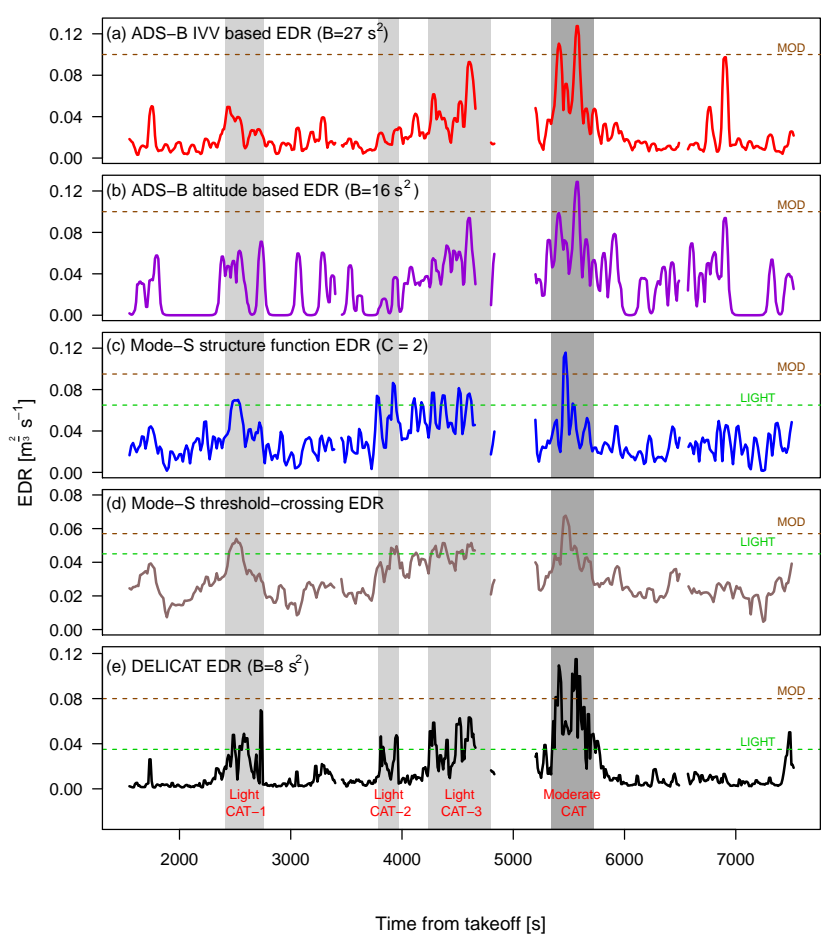

Figure 6. Results for FL9 for all analysed approaches to estimate EDR and a reference EDR (as in Fig. 2). The time series are scaled to match the intensity of the MOD event. For reference the indicative turbulence levels have been added to the plot. Those are reflecting the FL9 encounters and levels of noise inferred from the calm flights.

to modify the spectrum, thus causing the ADS-B-based estimates to have different values than the reference DELICAT EDR. Hence, normalizing to the same final range of values requires different choices of $B$.

The estimate based on the threshold crossing EDR displays values much smaller than those measured by the standard on-board sensors: $0.05 \mathrm{~m}^{\frac{2}{3}} \mathrm{~s}^{-1}$ for our estimate vs. $0.18 \mathrm{~m}^{\frac{2}{3}} \mathrm{~s}^{-1}$ for small business jet aircraft as reported in Sharman et al. (2014). Due to significant scatter of the reference results this may not be a serious issue. However, it is worth pointing out that our analysis is based on a theory developed for canopy turbulence considered in Poggi and Katul (2010) and not for the upper tropospheric/lower stratospheric turbulence investigated in the current analysis. The former is considered isotropic and homogeneous in measurement scales while the latter is often non-isotropic down to scales well below Mode-S EHS/ADS-B resolution and frequently intermittent (Cho et al., 2003). Thus, the constant quantity $7.5 \pi^{2}$ that can be found in Eq. (8) may differ significantly for UTLS turbulence.

The structure-function-based approach also results in a slight underestimation, when using $C=2$ in Eq. (5). The peak value of the recorded EDR in FL9 is $0.12 \mathrm{~m}^{\frac{2}{3}} \mathrm{~s}^{-1}$ for the borderline moderate case while the maximum value

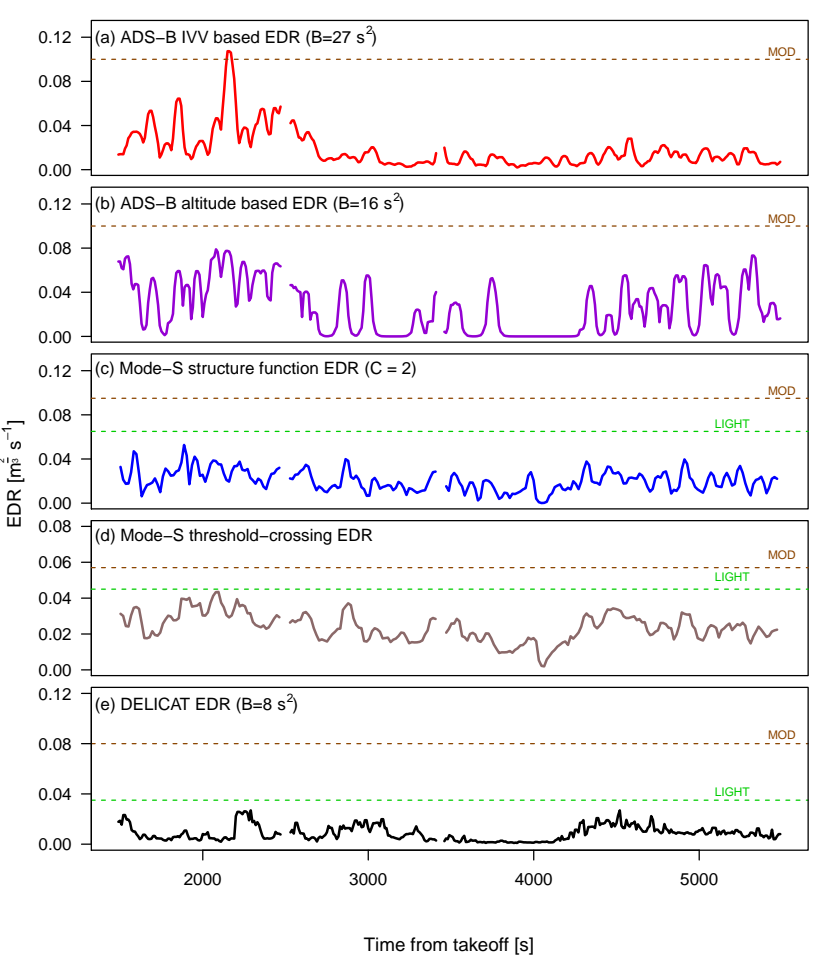

Figure 7. Results for FL8 for all analysed approaches to estimate EDR and a reference EDR (as in Fig. 2). The time series are scaled to match the intensity of the FL9 MOD event. For reference the indicative turbulence levels have been added to the plot. Those are reflecting the FL9 encounters and levels of noise inferred from the calm flights.

of EDR observed during light turbulence encounters was $0.09 \mathrm{~m}^{\frac{2}{3}} \mathrm{~s}^{-1}$. This is much closer to the PIREP-based values but still a little too low. In order to achieve EDR values corresponding exactly to the small business jet thresholds as reported in Sharman et al. (2014) one would have to reduce $C$ to 1 , which in turn is inconsistent with the fact that $C$ is known quite robustly to be approximately 2 over a large set of various flows (Sreenivasan, 1995).

Gaps in the time series visible in Figs. 6-8 are the result of discarding some flight fragments as unreliable. An unreliable flight fragment in our understanding is related to manoeuvres - either turn or significant ascent or descent. Those events will introduce (potentially large) errors. The observation was discarded if the roll off the aircraft exceeded $7^{\circ}$ in the $45 \mathrm{~s}$ neighbourhood of the observation or the $180 \mathrm{~s}$ moving average absolute value of the vertical velocity was greater than $1.3 \mathrm{~m} \mathrm{~s}^{-1}$. 


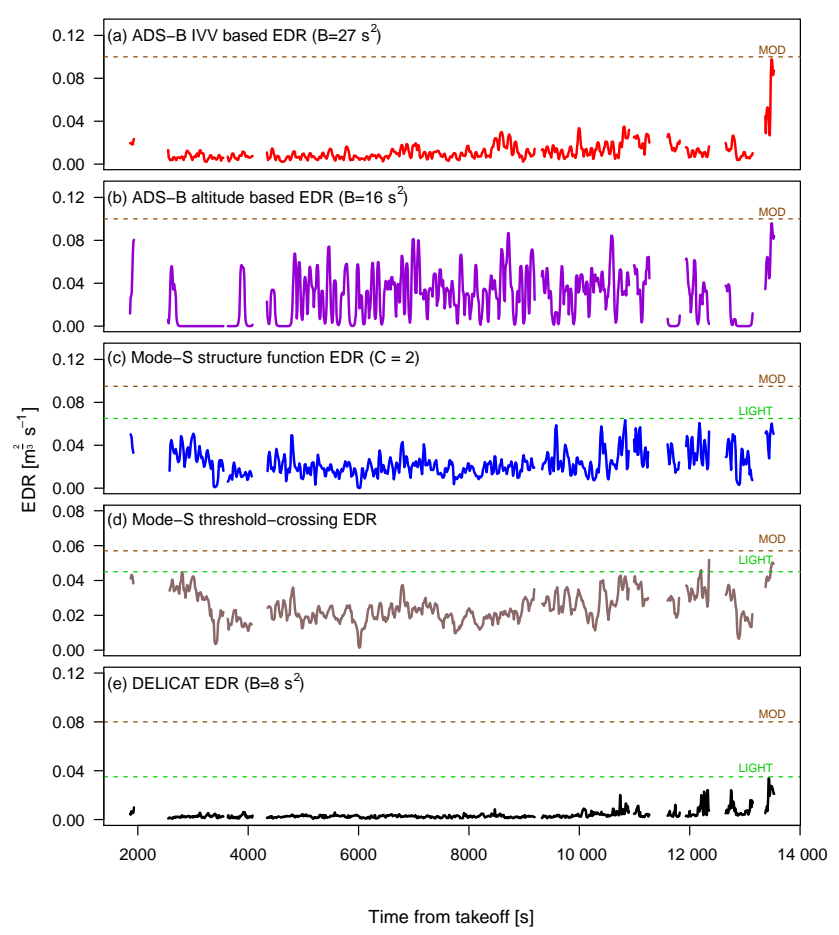

Figure 8. Results for FL6 for all analysed approaches to estimate EDR and a reference EDR (as in Fig. 2). The time series are scaled to match the intensity of the FL9 MOD event. For reference the indicative turbulence levels have been added to the plot. Those are reflecting the FL9 encounters and levels of noise inferred from the calm flights.

All of the methods display some potential in detecting turbulence. As indicated in Fig. 6, all of them have correctly identified the occurrence of borderline moderate clear-air turbulence (MOD event) during FL9. Other than that, there are significant differences. The ADS-B altitude-based approach suffers from a high level of background noise. This does not allow us to identify anything besides the single MOD event in FL9 simply because the light turbulence signal is drowned in the noise. The ADS-B IVV-based approach has much lower noise but it displays false MOD signal in calm air during flights FL9 and FL8. While FL9 false signal can be attributed to the aircraft manoeuvres (slight turn with quite sudden loss of altitude) there is no explanation recorded in the data for the FL8 issue. The wind-based Mode-S EHS methods displayed no such issues. There seems to be no significant false positive signal in any of the flights whilst all of the FL9 maxima were identified correctly by both methods. The noise levels in both cases were low enough for successful light turbulence recognition. However, both wind methods are much less precise in reflecting the location of the turbulence. Methods are characterized by an error of up to $100 \mathrm{~s}$ in peak turbulence displacement, which corresponds to approximately $25 \mathrm{~km}$ for the cruise speed of $250 \mathrm{~m} \mathrm{~s}^{-1}$. A concise summary of the es- timation methods as well as their performance is given in Table 2 .

\section{Discussion of the results}

We will begin our discussion with a parameter sensitivity analysis of the four analysed methods. In case of the ADSB-based methods it is clear from the Eq. (3) that there is only one free parameter - the time window for the moving variance calculation. This parameter is set to $30 \mathrm{~s}$. Higher values flatten the signal too much (acting not unlike an ill-gauged low-pass filter) while lower values cause too many spikes to arise making the signal illegible. In the reference publication (WMO, 2003) this period is set to $10 \mathrm{~s}$. In our case this value was too small due to quantization noise and filtering.

In case of the structure function method there is only one parameter - the period of flight time included in the single averaging. This parameter is now set to $30 \mathrm{~s}$. The lower values seem to be unreasonable (too low number of cases for averaging) while higher values act as an additional low-pass filter that reduces the signal.

The threshold crossing method has three free parameters: moving variance window, non-dimensional threshold value, and indicator function window. An indicator function window that is too small (less than $10 \mathrm{~s}$ ) causes stray peak signals to arise whereas much larger windows do not damp the signal. The indicator function windows as big as $360 \mathrm{~s}$ were tested and still showed quite clear response. In the end we have settled for the $60 \mathrm{~s}$ window. This quantity can still likely be tuned using more observations. The other parameters are much more critical for successful employment of this method. The moving variance window again acts like a low-pass filter when set to higher values. We have found out that $35 \mathrm{~s}$ is already too much. In our calculations this value has been set to $20 \mathrm{~s}$. The most critical parameter is the non-dimensional threshold $T_{C}$. The general behaviour is as expected: the bigger the modulus of $T_{C}$ the weaker the signal. However, if the absolute value of $T_{C}$ is too small the signal also becomes illegible. This is in accordance with observations of Poggi and Katul (2010). The disturbing feature of this method is that results for positive and negative $T_{C}$ are somewhat different. For positive $T_{C}$ the signal appears at 0.25 and quickly vanishes at 0.45 also the FL9 MOD event is quite weak also the period of calm air between Light CAT-2 and Light CAT-3 is not so well indicated (Fig. 9b). For negative thresholds the signal appears at -0.2 and disappears at -0.6 and the MOD event is more pronounced (Fig. 9a). This lack of symmetry is quite unexpected but there exists a range of absolute values of $T_{C}$ where, regardless of sign, the method still allows for detection of turbulence and for the correct discrimination of its intensity.

Another question is how good the constant TAS approximation for ADS-B-based methods is. DELICAT flights show up to $30 \%$ variance of TAS during cruise phases of the flights and one can expect this to influence the results significantly. 
Table 3. Summary of the EDR estimation approaches.

\begin{tabular}{lccc}
\hline Method & Performance & Advantages & Disadvantages \\
\hline ADS-B EDR & - IVV EDR: mostly satisfactory & - Global coverage & - Aircraft type dependent \\
& $\begin{array}{l}\text { but very significant false positives } \\
\text { found } \\
\text { - ALT EDR: significant noise, only }\end{array}$ & - Only one free parameter & - Performance issues (both ALT \\
& FL9 moderate identified & & and IVV EDR). \\
& Very good & - Potentially aircraft independent & - Coverage limited by SSR range \\
$\begin{array}{l}\text { Mode-S structure } \\
\text { function EDR }\end{array}$ & & - Only one free parameter & - Coverage limited by SSR range \\
$\begin{array}{l}\text { Mode-S threshold } \\
\text { crossing EDR }\end{array}$ & Good, some small issues in FL6 & - Potentially aircraft independent & - Three free parameters \\
\hline
\end{tabular}

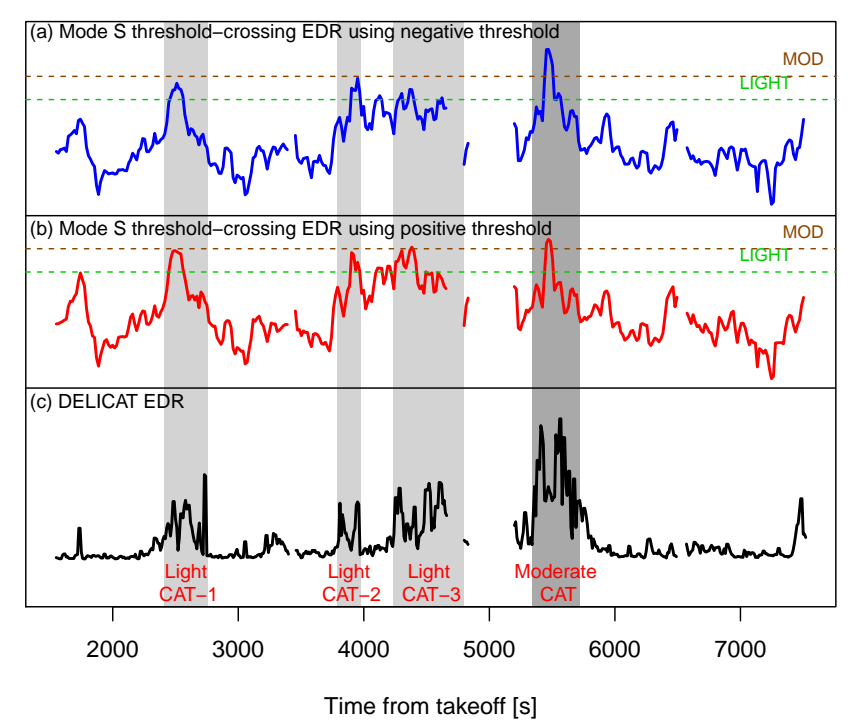

Figure 9. Comparison of the threshold crossing method results for $\left|T_{C}\right|=0.4$ for positive and negative threshold.

The comparison of the real TAS EDR estimates vs. the constant TAS EDR estimates shows that the application of the real TAS did not change much in any of the studied cases (see Fig. 10 for a FL9 example). In general this could be an issue, especially for the long-haul flights. However, in the present analysis the constant TAS approximation was satisfactory.

Another source of error can be the filtering. For the present paper we have selected the second-order Butterworth filters with expected frequencies that are based on turbulence physics. The upper bound $(10 \mathrm{~km})$ was introduced based on the previous studies and knowledge of turbulence scales (Lester, 1994; Frehlich and Sharman, 2010). The lower bound was driven by the limitations resulting from the signal discretization and sampling rates. Also we have used the Butterworth filter due to its relatively simple response characteristics. One can imagine a better suited filtering; however, we

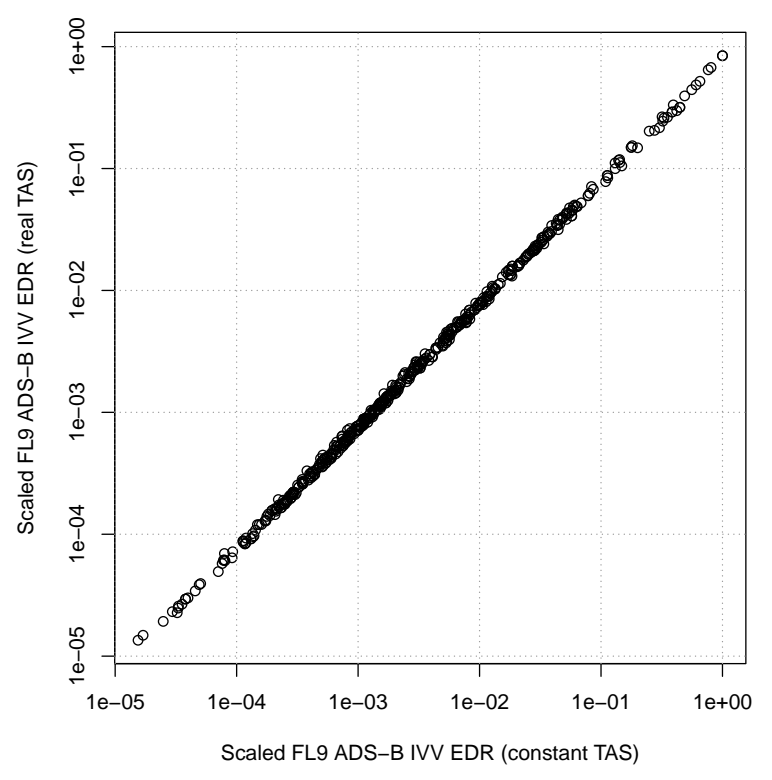

Figure 10. An exemplary comparison of EDR for FL9 calculated using IVV method using the real TAS record vs. the constant TAS approximation. We used $250 \mathrm{~m} \mathrm{~s}^{-1}$ as the constant TAS. Both of the EDR estimates were scaled to the range of values of constant TAS EDR thus are unitless and in approximate range $0-1$.

had too few cases at our disposal to refine the filtering as over-fitting would be the likely result.

It is also worthwhile to mention potential areas of use for these methods. All of the described methods allow a potential observer to use inexpensive equipment for observing upperair turbulence analogous to Haan et al. (2013) or Drüe et al. (2013). All of the methods use commercial aircraft as "sensors" so measurements in the regions not frequented by the aircraft are not feasible. Another limitation is the need for Mode-S/ADS-B transponders. Most newly produced aircraft are properly equipped. However, some of the older aircraft require to be retrofitted before they can provide the necessary data. The potential coverage is method dependent. ADS-B 
methods are based on automatic aircraft transmissions, thus allowing for potentially global reception. Mode-S EHS, however, is limited to the areas with the SSR Mode-S EHS coverage, which is in turn limited by the presence of the dedicated ground-based installations. In the end one must also consider that both of the ADS-B methods are heavily aircraft dependent, which is not visible in the analysis based on a single aircraft measurements, while the Mode-S EHS methods dependence on aircraft type should be limited since both Mode-S EHS methods are wind based.

\section{Conclusions}

We have proposed and made initial feasibility analysis of a set of four approaches aiming at estimating the turbulence using the commercial aircraft with help of the Mode-S and ADS-B protocols. Based on the available comparative material all of the methods have displayed some potential for finding turbulence. In the scope of our study the ADS-B-based methods perform much worse than the Mode-S EHS-based methods. The former allow only for a reliable recognition of a single FL9 borderline moderate case while the latter allow for quite faithful recreation of the turbulence presence and for differentiation into light and moderate categories.

Of the ADS-B methods the IVV-based calculation was better but we found that it is prone to display a very strong false signals, which would disqualify this method as a reliable source of measurements. In contrast, both of the Mode-S EHS methods performed almost flawlessly. We have compared the well-known structure function approach with a threshold crossing method that, to our best knowledge, has not been used in analysing upper-air data so far. Of those two the threshold crossing method requires more parameter fine tuning, yet the final performance of both methods was very similar.

Since the analysis presented here is only a feasibility check, it is necessary to conduct further research by employing a systematic comparison of the Mode-S EHS/ADS$B$ records against a reliable and proven source of turbulence measurements. For this purpose the in situ EDR data of Cornman et al. (1995) seem very appropriate. One just needs to identify a region where Mode-S EHS/ADS-B transmissions of an EDR broadcasting aircraft can be recorded and stored systematically. We are planning to run such validation over the Maastricht Upper Area Control Centre (MUAC) airspace sector in near future. The more comprehensive study may also help determine ways to overcome the main concerns about the practical use of the proposed methods namely potential aircraft dependence (mainly ADS-B), determination of better filtering, and treatment of missing/less regular data.

Also a great advantage of Mode-S EHS/ADS-B-based measurements is the ease, simplicity, and low price of the measurement set necessary to cover quite significant areas. One could even imagine that networks of such cheap receivers can be established. Another option is close cooper- ation with air navigation service providers who receive and store Mode-S EHS and ADS-B. However, another way of establishing observation network is cooperation with the existing communities such as flightradar24.com.

\section{Data availability}

The data used in this paper can be found in two sources. The reference Mode-S EHS/ADS-B data were supplied by the KNMI (Royal Netherlands Meteorological Institute). Use and distribution of these data is restricted by the appropriate KNMI policies. Access to these data can be granted by KNMI. The data collected by the DELICAT research aircraft can be accessed through the NLR (Netherlands Aerospace Centre). Use and distribution of these data is restricted by the appropriate NLR policies and the DELICAT grant agreement. Neither data set is currently publicly available due to the aforementioned restrictions.

Acknowledgements. The authors would like to thank the anonymous reviewers and J.-H. Kim for their comments. Jacek M. Kopeć would like to thank members of the DELICAT consortium (especially P. Vrancken and H. Veerman) for their helpful remarks on this paper. Jacek M. Kopeć's research was financed by National Science Centre, decision no. DEC-2013/11/N/ST10/00756. The paper uses data collected during the DELICAT campaign, which has received funding from the Seventh Framework Programme of the European Union (FP7/2007-2013) under grant agreement no. 233801. The data were collected by NLR in the Netherlands.

Edited by: A. Stoffelen

\section{References}

Cho, J. Y. N., Newell, R. E., Anderson, B. E., Barrick, J. D. W., and Thornhill, K. L.: Characterizations of tropospheric turbulence and stability layers from aircraft observations, J. Geophys. Res., 108, 8784, doi:10.1029/2002JD002820, 2003.

Cornman, L. B., Morse, C. S., and Cunning, G.: Real-time estimation of atmospheric turbulence severity from in-situ aircraft measurements, J. Aircraft, 32, 171-177, doi:10.2514/3.46697, 1995.

Drüe, C., Deimel, S., and Hoff, A.: A low-cost approach to derive upper-air wind measurements from ADS-B, 13th EMS Annual Meeting, Reading, UK, 9-13 September 2013, available at: http://presentations.copernicus.org/EMS2013-702_ presentation.pdf (last access: 21 August 2015), 2013.

Frehlich, R. and Sharman, R.: Climatology of velocity and tempera-ture turbulence statistics determined from rawinsonde and ACARS/AMDAR data, J. Appl. Meteorol. Clim., 49, 11491169, doi:10.1175/2010JAMC2196.1, 2010.

Gill, P. G. and Buchanan, P.: An ensemble based turbulence forecasting system, Meteorol. Appl., 21, 12-19, doi:10.1002/met.1373, 2014.

Grappel, R. D. and Wiken, R. T.: Guidance Material for Mode S-Specific Protocol Application Avionics, Tech. Rep. ATC-334, Lincoln Laboratory, MIT, Lexington, MA, 
USA, available at: https://www.ll.mit.edu/mission/aviation/ publications/publication-files/atc-reports/Grappel_2007_

ATC-334_WW-15318.pdf (last access: 7 August 2015), 2007.

Haan, S.: High-resolution wind and temperature observations from aircraft tracked by Mode-S air traffic control radar, J. Geophys. Res., 116, D10111, doi:10.1029/2010JD015264, 2011.

Haan, S., Haij, M., and Sondij, J.: The use of a commercial ADS$\mathrm{B}$ receiver to derive upper air wind and temperature observations from Mode-S EHS information in the Netherlands, Tech. Rep. TR-336, KNMI, De Bilt, the Netherlands, available at: http://www.knmi.n1/bibliotheek/knmipubTR/TR336.pdf (last access: 6 August 2015), 2013.

ICAO: Manual on Mode S Specific Services, Montreal, Canada, 2004.

ICAO: Annex 3 to the Convention on International Civli Aviation: Meteorological Service for International Air Navigation, International Civil Aviation Organisation, Montreal, Canada, 16th edn., 2007a.

ICAO: Annex 10 to the Convention on International Civli Aviation: Aeronautical Communications, International Civil Aviation Organisation, Montreal, Canada, 4th edn., 2007b.

ICAO: Guidance Material on Comparison of Surveillance Technologies (GMST), available at: http://www.icao.int/APAC/ Documents/edocs/cns/gmst_technology.pdf (last access: $12 \mathrm{Au}-$ gust 2015), 2007c.

James, P. K.: A review of radar observations of the troposphere in clear air conditions, Radio Sci., 15, 151-175, doi:10.1029/RS015i002p00151, 1980.

Kim, J.-H., Chan, W. N., Sridhar, B., and Sharman, R. D.: Combined winds and turbulence prediction system for automated airtraffic management applications, J. Appl. Meteorol. Clim., 54, 766-784, doi:10.1175/JAMC-D-14-0216.1, 2015.

Koch, S. E., Jamison, B. D., Lu, C., Smith, T. L., Tollerud, E. I., Girz, C., Wang, N., Lane, T. P., Shapiro, M. A., Parrish, D. D., and Cooper, O. R.: Turbulence and gravity waves within an upper-level front, J. Atmos. Sci., 62, 3885-3908, doi:10.1175/JAS3574.1, 2005.

Krozel, J. A. and Sharman, R. D.: Remote Detection of Turbulence via ADS-B, AIAA Guidance, Navigation, and Control Conference, AIAA 2015-1547, AIAA SciTech, doi:10.2514/6.20151547, 2015.

Lester, P.: Turbulence - A New Perspective for Pilots, Jeppesen Sanderson, Engelwood, CO, USA, 1994.

Meischner P., Baumann R., Höller H., and Jank, T.: Eddy Dissipation Rates in Thunderstorms Estimated by Doppler Radar in Relation to Aircraft In Situ Measurements, J. Atmos. Ocean. Tech., 18, 1609-1627, doi:10.1175\%2F15200426(2001)018\%3C1609\%3AEDRITE\%3E2.0.CO\%3B2, 2001.

Monin, A. S. and Yaglom, A. M.: Statistical Fluid Mechanics, vol. 2, MIT Press, Cambridge, MA, USA, 1975.

Mulally, D. J. and Anderson, A.: Correction of Aircraft Flux Valve Based Heading for Two-Dimensional Winds Aloft Calculations Using Weather Model Comparisons, 15th Symposium on Integrated Observing and Assimilation Systems for Atmosphere, Oceans, and Land Surface (IOAS-AOLS), 22-27 January 2011, Seattle, WA, USA, 2011.

NASA: U.S. Standard Atmosphere, National Aeronautics and Space Administration, Washington, USA, avail- able at: http://ntrs.nasa.gov/archive/nasa/casi.ntrs.nasa.gov/ 19770009539.pdf (last access: 13 August 2015), 1976.

NTSB: Review of U.S. Civil Aviation Accidents 2007-2009, Tech. Rep. NTSB/ARA-11/01, National Transportation Safety Board, Washington, USA, available at: www.ntsb.gov/investigations/ data/Documents/ARA1101.pdf (last access: 6 August 2015), 2011.

NTSB: Review of U.S. Civil Aviation Accidents 2010, Tech. Rep. NTSB/ARA-12/01, National Transportation Safety Board, available at: www.ntsb.gov/investigations/data/Documents/ ARA1201.pdf (last access: 6 August 2015), 2012.

Poggi, D. and Katul, G. G.: Evaluation of the turbulent kinetic energy inside canopies by zero- and level-crossing density methods, Bound.-Lay. Meteorol., 136, 219-233, doi:10.1007/s10546010-9503-2, 2010.

Signal developers: signal: Signal processing, available at: http: //r-forge.r-project.org/projects/signal/ (last access: 31 March 2016), 2013.

Sharman, R., Tebaldi, C., Wiener, G., and Wolff, J.: An integrated approach to mid- and upper-level turbulence forecasting, Weather Forecast., 21, 268-287, doi:10.1175/WAF924.1, 2006.

Sharman, R. D., Trier, S. B., Lane, T. P., and Doyle, J. D.: Sources and dynamics of turbulence in the upper troposphere and lower stratosphere: a review, Geophys. Res. Lett., 39, L12803, doi:10.1029/2012GL051996, 2012.

Sharman, R. D., Cornman, L. B., Meymaris, G., Pearson, J., and Farrar, T.: Description and derived climatologies of automated in situ eddy-dissipation-rate reports of atmospheric turbulence, J. Appl. Meteorol. Clim., 53, 1416-1432, doi:10.1175/JAMC-D13-0329.1, 2014.

Sreenivasan, K. R.: On the universality of the Kolmogorov constant, Phys. Fluids, 7, 2778-2784, doi:10.1063/1.868656, 1995.

Stone, E. K. and Pearce, G.: An operational network of Mode-S receivers for aircraft derived meteorological data, J. Atmos. Oceanic Technol., in press, doi:10.1175/JTECH-D-15-0184.1, 2016.

Trier, S. B. and Sharman, R. D.: Convection-permitting simulations of the environment supporting widespread turbulence within the upper-level outflow of a mesoscale convective system, Mon. Weather Rev., 137, 1972-1990, 2009.

Trier, S. B., Sharman, R. D., Fovell, R. G., and Frehlich, R. G.: Numerical simulation of radial cloud bands within the upper-level outflow of an observed mesoscale convective system, J. Atmos. Sci., 67, 2990-2999, doi:10.1175/2010JAS3531.1, 2010.

Trier, S. B., Sharman, R. D., and Lane, T. P.: Influences of moist convection on a cold-season outbreak of ClearAir Turbulence (CAT), Mon. Weather Rev., 140, 2477-2496, doi:10.1175/MWR-D-11-00353.1, 2012.

Veerman, H., Vrancken, P., and Lombard, L.: Flight testing delicat - a promise for medium-range clear air turbulence protection, in: European 46th SETP and 25th SFTE Symposium, Luleå, Sweden, 15-18 June 2014, available at: https://hal.archives-ouvertes. fr/hal-01111380 (last access: 7 August 2015), 2014.

Vrancken, P.: Airborne remote detection of turbulence with forward-looking LIDAR, in: Aviation Turbulence - Processes, Detection, Prediction, edited by: Sharman, R. D. and Lane, T. P., Springer, Cham, Switzerland, 2015. 
Williams, J.: Using random forests to diagnose aviation turbulence, Mach. Learn., 95, 51-70, doi:10.1007/s10994-013-53467, 2014.

Wyngaard, J. C.: Turbulence in the Atmosphere, Cambridge University Press, Cambridge, UK, 2010.
WMO: Aircraft Meteorological Data Relay (AMDAR) Reference Manual, World Meteorological Organization, WMO-No. 958, Geneva, Switzerland, 2003.

Wright, J. R. and Cooper, J. E.: Introduction to Aircraft Aeroelasticity and Loads, John Wiley and Sons, Chichester, UK, 2014. 